\title{
Nutritional Knowledge of Gastroenterology Fellows in the United States
}

\author{
Dina Halegoua-De Marzio*, Anastasia Shnitser and Stephanie Moleski \\ Division of Gastroenterology and Hepatology, Thomas Jefferson University, Philadelphia, PA
}

Received: November 26, 2013; Accepted: January 21, 2014; Published: January 23, 2014

"Corresponding author: Dina Halegoua-DeMarzio, Thomas Jefferson University Hospital, 132 S. 10th st. Rm 480 Philadelphia, PA 19148, USA, Fax: 215503-2146; E-mail: Dina.Halegoua-DeMarzio@jeffersonhospital.org

\begin{abstract}
Introduction: The aim of this study is to determine current nutritional knowledge and identify the perceived effectiveness of nutrition education among gastroenterology (GI) fellows.

Methods: A survey was created to collect demographic data, perceived nutritional knowledge, and a 20 question multiple choice examination (MCE) testing components of nutrition specific to GI. Adult GI fellowship coordinators at the 162 U.S. accredited adult fellowship programs were sent an email asking them to forward an internet link to their $1^{\text {st }}$ through $3^{\text {rd }}$ year GI Fellows where fellows could anonymously complete the survey.

Results: The survey was attempted by 137 fellows with 119 fellows fully completing it. The majority of fellows (86.6\%) wanted more nutrition focused education. Fellows perceived themselves as having the least knowledge in micro/macronutrients and providing nutritional support to patients. Fellows perceived themselves as having the strongest knowledge in technical skills to manage nutritional issues. The mean total score for MCE was $39.45 \%$ (standard deviation $(S D)=15.56)$. Fellows had the highest mean score in the questions related to nutritional support $(50.67 \%, \mathrm{SD}=21.55)$. The lowest mean score was on the subset of questions related to obesity $(26.08 \%$, SD $=13.01)$. Mean scores in other areas of nutrition included: nutrition assessment $46.45 \%$ ( $S D=14.18$ ), micro/macronutrients $34.07 \%$ (SD = 18.92), and nutrition in GI diseases $43.35 \%$ ( $S D=23.88)$.
\end{abstract}

Discussion: GI fellows believe their knowledge of nutrition is suboptimal and objective evaluation of nutrition knowledge confirms this belief. Formal nutrition education should be developed in the context of GI fellowship education.

Keywords: Nutrition; Graduate medical education; GI fellowship

\section{Introduction}

While important for all physicians in practice, a solid knowledge base regarding various aspects of nutrition and nutritional complications is critical for gastroenterologists and gastroenterology (GI) fellows. The American Gastroenterological Association (AGA) recognized the importance of nutrition when it formed the AGA Nutrition Curriculum Task Force in 1996 and created a revised nutrition curriculum in 2003 [1,2]. The Task Force's recommendation were that all GI fellows should acquire level 1 knowledge and all fellowship programs should provide the requisite training opportunities. The content areas in level 1 knowledge include basic nutrition principles as stated in Table 1 [2-4]. Despite the establishment of these guidelines, questions have been raised about the effectiveness of the current curriculum used in gastroenterology fellowships [5,6]. This may be in part due to a perceived lack of reimbursement for nutrition consultations and greater financial gain from performing procedure-based skills. Also, a lack of mentors and faculty trained in nutrition likely has been a considerable impediment to improved nutrition education [6]. A 2009 study focusing on the nutritional knowledge of Canadian GI fellows, found that most Canadian GI fellows did not receive any nutrition education during medical school and about one-third had not received any nutrition education during their fellowship [6]. Additionally, a 2013 study of Iranian GI fellows also found that most fellows had little or no nutrition education incorporated into their fellowship curriculum [7]. In both of these studies, GI fellows perceived their knowledge base in nutrition to be poor, with objective confirmation of this perception with multiple choice examination.

Gastroenterologists should receive effective formal nutrition training because it is a necessity in the everyday treatment of patients with gastrointestinal disease. Patients with gastrointestinal illnesses are prone to developing nutritional

Table 1: AGA task force recommendations on training in nutrition.

\begin{tabular}{|c|c|}
\hline 1 & $\begin{array}{c}\text { Basic principles of nutrient requirements, ingestion, digestion, } \\
\text { absorption, and metabolism in health and gastrointestinal disease }\end{array}$ \\
\hline 2 & $\begin{array}{r}\text { Assessment of nutrition status, including specific nutrient } \\
\text { deficiencies and excesses, protein energy, malnutrition, and obesity }\end{array}$ \\
\hline 3 & $\begin{array}{c}\text { Metabolic response to starvation and the pathophysiologic effects } \\
\text { of Malnutrition }\end{array}$ \\
\hline 4 & $\begin{array}{r}\text { Metabolic response to illness and injury and nutrient requirements } \\
\text { during stress states }\end{array}$ \\
\hline 5 & Indications for nutrition support \\
\hline 6 & $\begin{array}{c}\text { Implementation and management of nutrition therapy, including } \\
\text { modified diets, enteral tube feeding, and parental nutrition }\end{array}$ \\
\hline 7 & Pathophysiology and clinical management of obesity \\
\hline 8 & $\begin{array}{r}\text { Ethical and legal issues regarding provision and withdrawal of } \\
\text { nutrition support }\end{array}$ \\
\hline
\end{tabular}


abnormalities due to malabsorption, dietary restrictions, and altered nutrient requirements. Gastroenterologists must understand how to provide nutrition support, particularly appropriate indications, feeding route, formulation, and monitoring for both safety and efficacy. Additionally, obesity is becoming an increasingly prevalent chronic disease that may result in gastroenterological complications (i.e. nonalcoholic steatohepatitis).

The primary objective of this study was to assess the perceived nutritional knowledge and actual nutritional knowledge by surveying current adult GI fellows across the United States (U.S.) Specifically, we wished to investigate gastroenterology fellows' perceived needs for further education in the areas of nutrition assessment, nutrition support, macronutrient and micronutrient requirements, and obesity. Moreover, our goal was to determine GI fellows' baseline nutrition knowledge. With this information, methods could be identified to improve the effectiveness of current nutrition education among GI fellows.

\section{Methods \\ Participants}

A list of all 162 ACGME- accredited GI fellowship programs and the contact information of all of their fellowship coordinators were obtained from the American Gastroenterology Association (AGA) website. All fellowship coordinators were contacted via email and asked to forward an email containing a cover letter describing the study and an internet hyperlink to the SurveyMonkey ${ }^{\mathrm{TM}}$ website to all of their current GI fellows. On the website, participants anonymously completed the survey. If the fellows wished to provide their name and contact info, they were be entered to win one $\$ 100$ check card.

\section{Instrument}

The first section of the survey assessed needs and perceived knowledge of GI fellows in the 5 main areas of nutrition pertinent to GI education: (1) nutrition assessment (2) nutrition support (3) micronutrients and macronutrients (4) obesity and (5) nutrition as relevant to GI diseases. The items on this questionnaire employed a 5-point Likert Scale with options ranging from $1=$ strongly agree to $5=$ strongly disagree. This portion of the survey was drafted by 3 gastroenterologists at our institution based on the 5 domains of nutrition education identified by AGA Nutrition Curriculum Task Force in 2003 [2].

The second section of the questionnaire was a 20 item multiple choice examination (MCE) adapted and shortened from an examination that was previously validated and described in previous study by Raman, et al. [6]. The MCE is also based on the 5 main areas of nutrition pertinent to GI education and was used to assess actual nutrition knowledge of GI fellows. The following weighting scheme was assigned to each nutrition domain: nutrition assessment-20\%, nutrition support-20\%, obesity-20\%, micro/macronutrients-20\%, and nutrition in GI diseases-20\%. Fellows were asked to select the single best answer for each question. (See supplement for examination questions).

\section{Analysis}

Reliability of multiple choice examinations was calculated using Cronbach alpha. Results are expressed as mean and standard deviation (SD). Analysis of variance (ANOVA) was used to calculate differences between groups. This study received exemption approval from Thomas Jefferson University Institutional Review Board.

\section{Results \\ Demographics}

Of the approximately 1400 GI fellows in the U.S., 137 fellows attempted the survey (66.4\% males, 33.6\% females) with 119 fellows fully completing it with a response rate of $9.7 \%$ and $8.5 \%$, respectively. Postgraduate year of training was distributed equally among first, second, and third year fellows $(49,49$, and 39 fellows, respectively). In regards to medical education, 95.6\% of fellows have a medical doctorate (MD) degree and $4.4 \%$ had a doctorate of osteopathic medicine (DO). Location was divided based on census regions, $54.7 \%$ of fellows were from Northeast region, $27 \%$ Midwest, $10.9 \%$ West and $14.6 \%$ South. Only $16.1 \%$ of fellows reported any prior nutrition education before fellowship. When asked about nutrition education during fellowship, 34.3\% of fellows reported receiving no nutrition education and $65.7 \%$ reported receiving some nutrition education with the majority of those fellows $65.7 \%$ of fellows who reported receiving nutrition education, $76.3 \%$ reported receiving less then 4 hours of didactic lectures each fellowship year.

\section{Perceived Nutritional Knoweldge and Needs Assessment}

In evaluation of nutrition education needs assessment, 89.9\% of fellows reported wanting more nutrition education. Fellows were asked to rate their perceived level of knowledge in the domains of nutrition education mentioned above using a Likert scale (1-strongly disagree through 5-strongly agree). A total of $134 \mathrm{GI}$ fellows completed this portion of the survey. Fellows perceived themselves to have the least knowledge in nutrition support (i.e. use of oral, enteral and parenteral feeding) and macro/micronutrients with only $24.6 \%$ and $25.4 \%$ of fellows reporting they feel comfortable with these areas. In regards to other areas, fellows indicated that they had a greater knowledge base and comfort level in nutrition in GI disease at $47.7 \%$, obesity at $45.5 \%$, and nutritional assessment at $43.3 \%$ of GI fellows reporting that they felt comfortable.

\section{Multiple Choice Examination (MCE)}

Cronbach alpha of the MCE instrument was 0.59, indicating moderate internal consistency and reliability of the survey. Of the 137 fellows that attempted the survey, 119 fellows fully completed it. The mean total score for multiple choice examination was $39.4 \%$ (standard deviation (SD) = 15.5) (Figure 1). Fellows had the highest mean score in the questions related to nutritional support $(50.6 \%, \mathrm{SD}=21.5)$. The lowest mean score was on the subset of questions related to obesity $(26.0 \%, \mathrm{SD}=$ 13.0). Mean scores in other areas of nutrition included: nutrition assessment $46.4 \%$ (SD = 14.1), micro/macronutrients $34.0 \%$ (SD = 18.9), and nutrition in GI diseases $43.3 \%$ (SD = 23.8) (Figure 
1. Which of the following nutritional factors has been shown to BEST predict long term morbidity and/or mortality? (BMI $>30$ or $<18$ )

2. Which one of the following options represents historical features of the Subjective Global A ssessment? (W eight loss and gastrointestinal symptoms)

3. Which one of the following options represents potential complications of enteral nutrition? (A spiration and refeeding syndrome)

Questions 4 and 5 refer to the following stem: $Y$ ou are asked to see a 26 year old man with Crohn's disease. He has been admitted to hospital 3 days ago with a small bowel obstruction secondary to fibrostenotic disease of the terminal ileum. Prior to 3 days ago, his oral intake was normal despite ongoing abdominal pain and diarrhea related to his Crohn's disease. He has lost $2 \mathrm{~kg}$ since hospitalization, but his weight was stable prior to that. He is scheduled for surgery tomorrow for terminal ileal resection. His surgeon asks for your advice regarding nutrition support.

4. Would you start TPN today, expecting post-operative benefit? ( $\mathrm{No}$. He is young with no major comorbidities therefore at low risk of nutritional complications.)

5. W ould you recommend delaying surgery for TPN to improve outcome? (N o. Patients who are severely malnourished are most likely to benefit.)

6. Which of the following is NOT a clinical consequence of refeeding syndrome? (Hyperphosphatemia)

7. Which one of the following micronutrients is routinely added to TPN? (manganese)

8. In which of the following clinical situations should $>1.0 \mathrm{~g}$ protein per $\mathrm{kg}$ body weight be provided in nutrition support? (Patients with renal failure on hemodialysis)

9. Which one of the following gut hormones is responsible for promoting appetite? (G rehlin)

10. Which one of the following hormones plays an important role in inhibiting appetite? (Peptide YY)

11. Which of the following is NOT an adipocytokine? (G rehlin)

12. All of the following are manifestations of zinc deficiency EXCEPT? (parasthesias)

Questions 13 and 14 refer to the following stem: $Y$ ou are asked to assess a $50 \mathrm{yr}$ ma le with short bowel syndrome. This patient had a small bowel resection secondary to mesenteric ischemia 2 months ago, leaving him with $150 \mathrm{~cm}$ of residual small bowel and an end jejunostomy. He is now dependant on TPN 5 days per week. He is having $3 \mathrm{~L}$ of stool output per day. There is no evidence of enteric infection.

13. Which of the following pharmacological options is the most reasonable next therapeutic option? (Codeine $30 \mathrm{mg}$ po qid)

14. The patient above develops gradual onset of shortness of breath, 3-pillow orthopnea, and paroxysmal nocturnal dyspnea with mild ankle edema over a period of eight months. These symptoms may be a result of which of the following? (Selenium deficiency)

15. When does MAXIM AL small bowel adaptation occur post small bowel resection? (18 months)

16. Which of the following foods does not contain gluten and is acceptable for patients with celiac disease to consume? (Rice)

17. A 35 year old morbidly obese female underwent vertical band gastroplasty (VBG) 6 months ago. She has lost $50 \mathrm{lbs}$ of weight over 6 months. She is now complaining of 6-8 loose watery bowel movements per day ongoing for the past 2 months. Prior to 2 months ago she was having 1 formed bowel movement per day for years. Which of the following statements regarding this scenario is TRUE? (The diarrhea is not related to the VBG and requires a full work-up)

18. Which of the following statements regarding protein intake in patients with chronic liver disease is TRUE? (Protein restriction should NOT be considered routinely in patients with chronic liver disease)

19. Which of the following statements regarding macronutrient energy value is TRUE? (Protein provides $4 \mathrm{kcal} / \mathrm{gram}$ consumed)

20. A product lists the following nutrition information: Serving size $90 z$ Servings per package 1 Calories 240 Protein $19 \mathrm{~g}$ Carbohydrate $19 \mathrm{~g}$ Fat $10 \mathrm{~g}$ W hat is the percentage of calories provided by fat in this product? $(38 \%)$

$62.8 \%$

$51.3 \%$

$69.0 \%$

$15.0 \%$

$33.6 \%$

$61.1 \%$

$15.5 \%$

$16.8 \%$

$42.5 \%$

$31.9 \%$

$13.3 \%$

$31.9 \%$

$49.6 \%$

$60.2 \%$

$29.2 \%$

$55.8 \%$

$19.5 \%$

$75.2 \%$

$42.5 \%$

Figure 1: Multiple Choice Examination Questions with Percentages. Correct answers to each question are listed parenthetically. Percentages indicate the proportion of fellows who provided correct answers

2). Analysis of variance (ANOVA) confirmed that there was no difference $(\mathrm{p}<0.93)$ in the mean scores for fellows depending on their level of training.

\section{Discussion}

This is the largest national survey of GI fellow nutritional knowledge of in the U.S. The main finding this study is that effective nutrition education is lacking from the curriculum of many GI fellowship programs, despite being one of the core competencies of GI education. The majority of fellows did not feel that their nutrition knowledge was at an adequate level which is consistent with their below average scores of the MCE. Although nutrition knowledge is lacking, the overwhelming majority of fellows where interested in learning more about nutrition which 


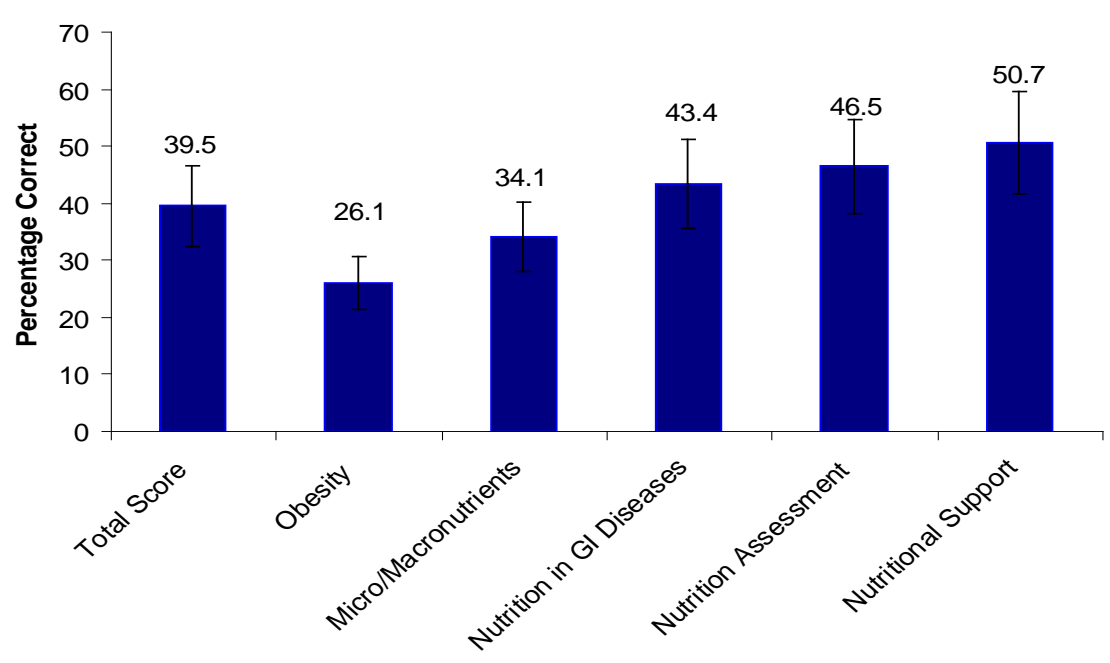

Figure 2: Multiple choice examination total test and subscale percentages.

means that this lack of knowledge is possibly correctable with appropriate curriculum development.

In a study from Mayo Clinic in 2005, they found similar deficiencies in nutrition education among GI fellowship programs [4]. This study also consisted of a course with 20 hours of nutrition education. After this course, fellows did significantly better on their post course nutrition based MCE. Unfortunately, since the publication of this study in 2005, the knowledge base and comfort level in the area of nutrition appears to have not improved among GI fellows. One must hypothesize that the established AGA nutrition curriculum guidelines and core competencies are not being fulfilled in most programs.

As stated earlier, our study is very similar to a nutrition survey study of Canadian GI fellows completed by Raman et al. in 2009 [6]. In our present study, GI fellows did particularly poorly on the questions concerning micronutrients/macronutrients and obesity on the MCE. Micro/macronutrients and obesity were also the lowest scored areas in the study by Raman et al. and Eslamian et al. [6,7]. The scores from that study in these areas were $37.8 \%$ and $40.1 \%$ for micro/macronutrients and obesity, respectively. These particular areas should be emphasized when developing nutrition curriculum in the future.

The major limitation of the study is the small sample size with only $\sim 10 \%$ of GI fellows in the U.S responding to the study. Another limitation includes the anonymous online nature of the study. Due to this anonymity, it can not be determined if the demographic information given by respondents is correct. In addition, references may have been used by the fellows to answer the knowledge questions and fellows with more interest in nutrition may have more likely to respond to the survey. Population bias is also a possible limitation. Fellows with more interest in nutrition may have been more likely to respond to the survey. Generalization of the results therefore must be carried out with caution and the results may not represent the knowledge base or attitudes of all GI fellows.
In conclusion, we have shown that there is a perception that nutrition knowledge among GI fellows in the U.S. appears to be suboptimal; objective evaluation of nutrition knowledge in this cohort confirms the legitimacy of these beliefs as evidenced by poor overall performance on a nutrition knowledge examination. On the basis of our results, the current process of allowing fellowship programs to design their own methods of teaching nutrition is not successful. Formal nutrition education should be developed in the context of GI fellowship education with measures of competency. This level of competency needs to be achieved before completion of GI fellowship.

\section{Acknowledgements}

Maitreyi Raman, MD, Claudio Violato, PhD, and Sylvain Coderre, MD for allowing adaptation of their previously studied multiple choice examination for this study. Financial Support was offered by Thomas Jefferson University Hospital Division of Gastroenterology and Hepatology.

\section{References}

1. Task Force on Training in Nutrition (1996) American gastroenterological association. Gastroenterology 110: 1293-1295.

2. Task Force on Training in Nutrition (2003) American Gastroenterological Association. Gastroenterology 124: 1085-1087.

3. Heimburger DC (2002) Training and Certifying Gastroenterologists as Physician Nutrition Specialists. J Clin Gastroenterol 34(5): 505-508.

4. Scolapio JS, Buchman AL, Floch M (2008) Education of gastroenterology trainees, first annual fellows' nutrition course. J Clin Gastroenterol 42: 122-127.

5. Singh H, Duerksen DR (2006) Survey of clinical nutrition practices of Canadian gastroenterologists. Can J Gastroenterology 20: 527-530.

6. Raman M, Violato C, Coderre S (2009) How Much do Gastroenterology Fellows Know About Nutrition? J Clin Gastroenterol 45(6): 559-564.

7. Eslamian G, Jacobson K, Hekmatdoost A (2013) Clinical Nutrition Knowledge of Gastroenterology Fellows: Is There Anything Omitted? Acta Med Iran 51(9): 633-37. 